Published in final edited form as:

Gastroenterology. 2014 June ; 146(7): 1659-1668. doi:10.1053/j.gastro.2014.02.054.

\title{
Loss-of-function of the Voltage-gated Sodium Channel NaV1.5 (Channelopathies) in Patients with Irritable Bowel Syndrome
}

\author{
Arthur Beyder $^{\# 1}$, Amelia Mazzone ${ }^{\# 1}$, Peter R. Strege ${ }^{\# 1}$, David J. Tester ${ }^{\# 2}$, Yuri A. Saito ${ }^{1}$, \\ Cheryl E. Bernard ${ }^{1}$, Felicity T. Enders ${ }^{3}$, Weronica E Ek ${ }^{4}$, Peter T Schmidt ${ }^{5}$, Aldona \\ Dlugosz $^{5}$, Greger Lindberg ${ }^{5}$, Pontus Karling ${ }^{6}$, Bodil Ohlsson ${ }^{7}$, Maria Gazouli ${ }^{8}$, Gerardo \\ Nardone $^{9}$, Rosario Cuomo ${ }^{10}$, Paolo Usai-Satta ${ }^{11}$, Francesca Galeazzi ${ }^{12}$, Matteo Neri ${ }^{13}$, \\ Piero Portincasa ${ }^{14}$, Massimo Bellini ${ }^{15}$, Giovanni Barbara ${ }^{16}$, Michael Camilleri ${ }^{1}$, G. Richard

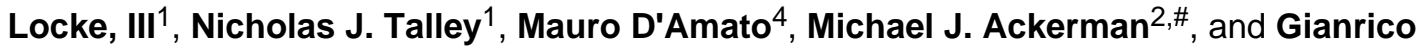 \\ Farrugia $^{1, \#}$ \\ ${ }^{1}$ Enteric Neuroscience Program, Division of Gastroenterology \&Hepatology, Department of \\ Physiology \& Biomedical Engineering, Mayo Clinic, Rochester, Minnesota
}

2Departments of Medicine (Cardiovascular Diseases), Pediatrics (Pediatric Cardiology), and Molecular Pharmacology \& Experimental Therapeutics and the Windland Smith Rice Sudden Death Genomics Laboratory, Mayo Clinic, Rochester, Minnesota;

${ }^{3}$ Division of Biomedical Statistics and Informatics, Department of Health Sciences Research, Mayo Clinic, Rochester, Minnesota

\footnotetext{
\#Corresponding authors: Gianrico Farrugia, MD and Michael J. Ackerman MD, PhD Mayo Clinic 200 First Street SW, Rochester, Minnesota 55905 Phone: 507-284-4695. Fax: 507-284-0266. farrugia.gianrico@mayo.eduackerman.michael@mayo.edu.

Publisher's Disclaimer: This is a PDF file of an unedited manuscript that has been accepted for publication. As a service to our customers we are providing this early version of the manuscript. The manuscript will undergo copyediting, typesetting, and review of the resulting proof before it is published in its final form. Please note that during the production process errors may be discovered which could affect the content, and all legal disclaimers that apply to the journal pertain.

Disclosures: MJA: 1) Consultant - Boston Scientific, Medtronic, and St. Jude Medical; 2) Other/Royalties - Transgenomic (FAMILION). The remaining authors have no disclosures
}

Author Contributions:

AB - study concept and design, analysis and interpretation of data, drafting of the manuscript, critical revision of the manuscript for important intellectual content, statistical analysis; AM -acquisition of data, analysis and interpretation of data, critical revision of the manuscript for important intellectual content, statistical analysis; PRS - study concept and design, acquisition of data, analysis and interpretation of data, drafting of the manuscript, critical revision of the manuscript for important intellectual content, statistical analysis; DJT - study concept and design, acquisition of data, analysis and interpretation of data, statistical analysis; YAS - study concept and design, analysis and interpretation of data, critical revision of the manuscript for important intellectual content, statistical analysis; CEB - acquisition of data; FTE - analysis and interpretation of data, critical revision of the manuscript for important intellectual content, statistical analysis; WEE - analysis and interpretation of data, critical revision of the manuscript for important intellectual content, statistical analysis; PTS - acquisition of data, analysis and interpretation of data; AD - acquisition of data, analysis and interpretation of data; GL -acquisition of data, analysis and interpretation of data; $\mathbf{P K}$ - acquisition of data, analysis and interpretation of data; BO - acquisition of data, analysis and interpretation of data; MG -acquisition of data, analysis and interpretation of data; GN - acquisition of data, analysis and interpretation of data; RC - acquisition of data, analysis and interpretation of data; PUS -acquisition of data, analysis and interpretation of data; FG - acquisition of data, analysis and interpretation of data; MN - acquisition of data, analysis and interpretation of data; $\mathbf{P P}$-acquisition of data, analysis and interpretation of data; $\mathbf{M B}$ - acquisition of data, analysis and interpretation of data; GB - acquisition of data, analysis and interpretation of data; $\mathbf{M C}$ - study concept and design, critical revision of the manuscript for important intellectual content; GRL - study concept and design, critical revision of the manuscript for important intellectual content; NJT - study concept and design, critical revision of the manuscript for important intellectual content; MDA - study concept and design, analysis and interpretation of data, critical revision of the manuscript for important intellectual content, statistical analysis, obtained funding; MJA -study concept and design, critical revision of the manuscript for important intellectual content, obtained funding, administrative, technical, or material support, study supervision; GF study concept and design, analysis and interpretation of data, drafting of the manuscript, critical revision of the manuscript for important intellectual content, statistical analysis, obtained funding, administrative, technical, or material support, study supervision. 
${ }^{4}$ Department of Biosciences and Nutrition, Karolinska Institutet, Stockholm, Sweden

${ }^{5}$ Department of Gastroenterology and Hepatology, Karolinska University Hospital, Karolinska Institutet, Stockholm, Sweden

${ }^{6}$ Department of Medicine, Umeå University, Umeå, Sweden

${ }^{7}$ Department of Clinical Sciences, Skånes University Hospital, Malmoe, Sweden

${ }^{8}$ Laboratory of Biology, School of Medicine, University of Athens, Athens, Greece

${ }^{9}$ Gastroenterology Unit, Department of Clinical Medicine and Surgery, University Federico II, Naples, Italy

${ }^{10}$ Digestive Motility Diseases, Department of Clinical Medicine and Surgery, Federico II University Hospital , Naples, Italy

${ }^{11}$ S.C. Gastroenterologia, Azienda Ospedaliera G. Brotzu, Cagliari, Italy

${ }^{12}$ UOC Gastroenterologia, Padova University Hospital, Padova, Italy

${ }^{13}$ Department of Medicine and Aging Sciences and CESI, G. D'Annunzio University \& Foundation, Chieti, Italy

${ }^{14}$ Department of Biomedical Sciences and Human Oncology (DIMO), Clinica Medica 'A. Murri', University of Bari Medical School, Bari, Italy

${ }^{15}$ Gastroenterology Unit, Department of Gastroenterology, University of Pisa, Pisa, Italy

${ }^{16}$ Department of Medical and Surgical Sciences, University of Bologna, St. Orsola - Malpighi Hospital, Bologna, Italy

\# These authors contributed equally to this work.

\section{Abstract}

BACKGROUND \& AIMS-SCN5A encodes the a-subunit of the voltage-gated sodium channel $\mathrm{Na}_{V}$ 1.5. Many patients with cardiac arrhythmias caused by mutations in SCN5A also have symptoms of irritable bowel syndrome (IBS). We investigated whether patients with IBS have $S C N 5 A$ variants that affect the function of Nav1.5.

METHODS-We performed genotype analysis of SCN5A in 584 persons with IBS and 1380 without (controls). Mutant forms of SCN5A were expressed in HEK-293 cells, and functions were assessed by voltage clamp analysis. A genome-wide association study (GWAS) was analyzed for an association signal for the SCN5A gene, and replicated in 1745 patients in 4 independent cohorts of IBS patients and controls.

RESULTS-Missense mutations were found in SCN5A in 13/584 patients (2.2\%, probands). Diarrhea-predominant IBS (IBS-D) was the most prevalent form of IBS in the overall study population (25\%). However, a greater percentage of individuals with SCN5A mutations had constipation-predominant IBS (IBS-C, 31\%) than IBS-D (10\%, $P<.05)$. Electrophysiologic analysis showed that 10/13 detected mutations disrupted $\mathrm{Na}_{V} 1.5$ function ( 9 reduced and 1 increased function); p.A997T-Nav1.5 had the greatest effect in reducing $\mathrm{Na}_{\mathrm{V}} 1.5$ function. Incubation of cells that expressed this variant with mexiletine restored their sodium current; 
administration of mexiletine to 1 carrier of this mutation (who had IBS-C) normalized their bowel habits. In the GWAS and 4 replicated studies, the SCN5A locus was strongly associated with IBS.

CONCLUSIONS-About $2 \%$ of patients with IBS carry mutations in SCN5A. Most of these are loss-of-function mutations that disrupt $\mathrm{Na}_{\mathrm{V}} 1.5$ channel function. These findings provide a new pathogenic mechanism for IBS and possible treatment options.

\section{Keywords}

genetics; GI motility; voltage-gated sodium channel; polymorphism

\section{Introduction}

Irritable bowel syndrome (IBS) is a highly prevalent disorder affecting $15-20 \%$ of the western world population. ${ }^{1}$ IBS pathophysiology involves abnormalities in gastrointestinal (GI) motility and visceral sensory processing. ${ }^{2}$ Familial aggregation ${ }^{3}$ and twin studies ${ }^{4}$ suggest that genetics play a role in IBS. Genotyping supports this notion, but specific impact of individual genes remains unclear. ${ }^{5}$ Ion channels are excellent pathophysiologic and therapeutic targets because they are directly involved in both GI motility ${ }^{6,7}$ and visceral pain. ${ }^{8}$ Therefore, ion channelopathies may cause IBS in some cases.

Voltage-gated sodium channels $\left(\mathrm{Na}_{\mathrm{V}}\right)$ are present in the gastrointestinal smooth muscle, including rat fundus, ${ }^{9}$ human and canine jejunum, ${ }^{10}, 11$ rat and human colon. ${ }^{12} \mathrm{~A}$ particular tetrodotoxin-resistant sodium channel Nav1.5 (encoded by SCN5A) is expressed in the human smooth muscle cells and interstitial cells of Cajal (ICC) of the small intestine and colon. ${ }^{13,14}$ Among other functions, ICC are GI pacemakers that generate cyclical depolarizations (slow waves) transmitted to the smooth muscle to provide the electrical stimulus for contraction. ${ }^{15}$ In human GI smooth muscle, Nav channels appear to be excitatory for slow waves ${ }^{14}$ and $\mathrm{Na}_{\mathrm{V}} 1.5$ is functionally relevant in the human GI tract, as pharmacologic block of $\mathrm{Na}_{\mathrm{V}} 1.5$ is associated with constipation. ${ }^{16}$

SCN5A is also expressed densely in human cardiomyocytes. ${ }^{17}$ SCN5A rare mutations ${ }^{18}$ and common variants ${ }^{19}$ are associated with cardiac arrhythmias. Interestingly, patients with arrhythmia-predisposing mutations in SCN5A have more gastrointestinal symptoms and increased prevalence of IBS when compared to patients with other arrhythmia-related ion channelopathies. ${ }^{20}$ Conversely, a subset of patients with IBS may have SCN5A mutations despite a normal cardiac phenotype. In a pilot study, a rare $S C N 5 A$ missense mutation was found in a patient with IBS and no cardiac conduction abnormalities. This mutation resulted in Nav1.5 channels with decreased peak currents and mechanosensitivity. ${ }^{21}$ In the present study, we screened large cohorts of IBS patients to determine the prevalence of SCN5A polymorphisms and mutations in IBS, tested whether the identified mutations led to altered $\mathrm{Na}_{V} 1.5$ function, and successfully treated an IBS-C patient with the SCN5A mutation resulting in the most severe electrophysiology abnormalities. These data may represent a novel pathophysiologic mechanism and provide new therapeutic options for a subset of IBS patients. 


\section{Materials and Methods}

\section{Subjects}

Mayo Clinic Institutional Review Board (IRB) approved the study. The mutation discovery cohort included patients ( $\mathrm{n}=584)$ aged 18-69 years recruited between February 2004 and July 2005 at the Mayo Clinic Rochester. The cohort used for replication of an independent genome wide association study (GWAS) for SCN5A $(\mathrm{n}=1745)$ includes additional patients from United States (Mayo Clinic), and multi-center cohorts from Sweden, Italy and Greece and detailed in Supplementary Methods.

\section{Genetic Analysis of SCN5A}

Genetic analysis was previously described ${ }^{22}$ and GWAS data and analysis are both detailed in Supplementary Methods.

\section{Expression Vector Construction and HEK-293 Cell Transfection}

Previously described ${ }^{21}$ site-directed mutagenesis and co-transfection (pEGFP-C1 and channel constructs) into HEK-293 cells are detailed in Supplementary Methods.

\section{Whole Cell Electrophysiology}

Solutions and whole cell voltage clamp setup are previously described ${ }^{21}$ and detailed in Supplementary Methods. Voltage-clamp protocols were designed de-novo for this study and are described in detail in Supplementary Methods.

\section{Voltage-Clamp Protocols}

\section{Definition of Electrophysiologic Loss- (LOF) and Gain-of-Function (GOF)-}

LOF was defined as a decrease in overall $\mathrm{Na}^{+}$charge flux that would result from a decrease in peak current $\left(\mathrm{I}_{\text {peak }}\right)$, slowed time to peak $\left(\mathrm{t}_{\text {peak }}\right)$, depolarization (right-shift) in the halfpoint of the voltage-dependence of activation $\left(\mathrm{V}_{1 / 2 \mathrm{a}}\right)$, and decrease in slope of the voltagedependence of activation $\left(\delta \mathrm{V}_{\mathrm{a}}\right)$; or as a hyperpolarization (left-shift) of the half-point of the voltage-dependence of inactivation $\left(\mathrm{V}_{1 / 2 \mathrm{i}}\right)$, decrease in slope of the voltage dependence of inactivation $\left(\delta \mathrm{V}_{\mathrm{i}}\right)$, and decrease of the time constants of inactivation $\left(\tau_{\mathrm{s}}, \tau_{\mathrm{f}}\right)$. GOF was defined as an increase in overall $\mathrm{Na}^{+}$charge flux with changes opposite of those described above for loss-of-function.

\section{Clinical Case of Mexiletine Treatment of an A997T-Nav1.5 Patient with IBS-C-} The study team carried out a prospective open-label study on the effect of mexiletine on bowel habits and whole gut transit with 48-hour colon transit measurements completed at baseline and after the 5-day treatment period (Clinicaltrials.gov NCT01717404). Details are in Supplementary Methods.

\section{Results}

\section{IBS Patient Cohort}

The characteristics of the mutation-discovery cohort are shown in Error! Reference source not found.. The median age was 49.5 years; they were predominately Caucasian (94\%) 
females (83\%). Diarrhea-predominant IBS (IBS-D, 25\%) was more common than constipation-predominant IBS (IBS-C, 10\%, Error! Reference source not found.). The remainder of the cohort was mixed IBS (IBS-M, 31\%) and those who did not classify by our questionnaires. IBS patients from the GWAS-replication cohorts had similar characteristics (Supplementary Table 4).

\section{IBS Subjects with SCN5A variations and confirmation of the findings in a GWAS}

Thirteen of the 584 (2.2\%) subjects had unique SCN5A amino acid altering missense mutations (Figure 1). The 13 missense mutations were not observed in 2760 reference alleles. The demographics of the subjects with SCN5A mutations (probands) were no different from the cohort described above (Error! Reference source not found.). However, unlike the IBS patient cohort, the probands were more often IBS-C (31\%) than IBS-D (10\%, $\mathrm{P}<0.05)$ (Error! Reference source not found.). This subset of IBS subjects with a SCN5A mutation had normal QTc $(424 \pm 22 \mathrm{~ms})$ and PR $(164 \pm 42 \mathrm{~ms})$ intervals. All ECGs were reviewed and while electrophysiologic abnormalities were discovered none were formally diagnostic (detailed in Supplementary Information). There were also nine distinct polymorphisms detected in 17 (2.9\%) subjects. All polymorphisms had been previously characterized and shown to have electrophysiologic abnormalities (Supplementary Table 3).

To independently evaluate the association of SCN5A polymorphisms with IBS we inspected data from a Swedish genome-wide association study (GWAS) of IBS, and an association signal of nominal significance was detected for SCN5A (Supplementary Figure 3). We followed-up this signal by genotyping 17 SCN5A single nucleotide polymorphisms (SNPs) in 1745 additional individuals from 4 independent IBS cohorts from Sweden, Italy, Greece and US. Several gave rise to stronger association in a meta-analysis of GWAS and replication data (Supplementary Table 5).

\section{Molecular Characteristics of the Identified Nav1.5 Mutations}

$S C N 5 A$ encodes $\mathrm{Na}_{V} 1.5$, a 2016-amino acid transmembrane protein with four homologous domains (DI-DIV) of six transmembrane segments each. One of the 13 identified mutations localized to the $\mathrm{N}$-terminus, four to the $\mathrm{C}$-terminus, six resided in the inter-domain linkers (IDL) and two in the transmembrane segments of DI and DIII (Figure 1). Six mutations had been previously associated with cardiac conduction pathologies: four were associated with Brugada type 1 (A997T, T220I, G615E, P648L), ${ }^{23}$ two with long-QT type 3 (G615E ${ }^{24,} 25$, $\left.\mathrm{T} 1304 \mathrm{M}^{26}\right)$, one with sudden infant death syndrome (T1304M $\left.\mathrm{M}^{27,28}\right)$, one with sick sinus syndrome $\left(\mathrm{T} 220 \mathrm{I}^{29,30}\right)$, and one with sudden death in women $\left(\mathrm{G} 615 \mathrm{E}^{31}\right)$. The other seven mutations were novel (I94V, T630M, G1158S, R1512Q, E1780G, A1870D, L1896V, and M1952T). However, even for the previously identified mutations, limited functional data were available for only three: T220I, ${ }^{29,}{ }^{30} \mathrm{G} 615 \mathrm{E}^{31}$ and T1304M. ${ }^{27}$

\section{Electrophysiologic Examination of Nav1.5 Mutations}

All 13 missense mutations were inserted by site-directed mutagenesis into the most common SCN5A background H558/Q1077del (WT), transfected into HEK-293 cells, and examined by whole cell voltage clamp electrophysiology. We explored the functional aspects of $\mathrm{Na}_{V} 1.5$ that could contribute to GI electrophysiology. 
Ten of the 13 missense mutations (77\%) showed functional differences when compared with wild-type controls (Error! Reference source not found., Figure 2). Abnormally functioning mutations included one in a transmembrane segment (T220I), 5 in IDL1 (G615E, T630M, P648L), 2 in IDL2 (A997T, G1158S), and 2 in the C-terminus (E1780G, L1896V). No statistically significant abnormalities were found in three mutations (T1304M, R1512Q, and M1952T).

Functional abnormalities were found in two-thirds of all the tested parameters (Error! Reference source not found.). To generalize the pattern of the discovered abnormalities, we separated functional abnormalities into loss-of-function (LOF, blue in Error! Reference source not found.) or gain-of-function (GOF, red in Error! Reference source not found.). Using this classification, 19/21 (90\%) of abnormal parameters were LOF and 2/21 (10\%) abnormal parameters were GOF. Overall, 9/10 (90\%) mutations with electrophysiologic abnormalities were LOF and one (10\%) was a GOF (Error! Reference source not found.).

Steady-state properties ( $\mathrm{I}_{\text {peak }}, \mathrm{V}_{1 / 2 \mathrm{a}}, \delta \mathrm{V}_{\mathrm{a}}, \mathrm{V}_{1 / 2 \mathrm{i}}, \delta \mathrm{V}_{\mathrm{i}}$ ) were affected for 6 mutations. There was a dramatic decrease in peak current $\left(\mathrm{I}_{\text {peak }}\right)$ for A997T (Figure 2A). For three mutations (T220I, G615E, A997T) there were significant shifts in voltage-dependence of activation and/or inactivation which would predict a decrease in the density and availability of $\mathrm{Na}^{+}$ current (Figure 2B). Decrease in the slope of the voltage-dependence of inactivation $\left(\delta \mathrm{V}_{\mathrm{i}}\right)$ was the most commonly affected parameter ( 6 of 13 mutations). The shifts in the half-points of voltage-dependence $\left(\mathrm{V}_{1 / 2 \mathrm{a}}\right.$ and $\left.\mathrm{V}_{1 / 2 \mathrm{i}}\right)$ and decrease in slopes of the voltage-dependence of activation and inactivation $\left(\delta \mathrm{V}_{\mathrm{a}}\right.$ and $\left.\delta \mathrm{V}_{\mathrm{i}}\right)$ resulted in significant decreases in the window current (area under the curves in Figure 2C). As window currents are steady-state currents near resting potential, smaller window currents may decrease $\mathrm{Na}^{+}$influx and result in hyperpolarization.

Significant changes in kinetic properties were found for 6 mutations, with 3 affecting kinetics of activation (3/4 LOF, Figure 2D) and 3 affecting kinetics of inactivation (all 3/3 LOF, Figure 2E). Time to peak ( $\mathrm{t}_{\text {peak }}$ ) was significantly slower for three mutations and faster for L1896V (Figure 2A). Interestingly, given the slower GI kinetics compared to the heart, while the faster of two inactivation time constants $\left(\tau_{f}\right)$ was not affected for any of the mutations, the slower time constant $\left(\tau_{\mathrm{s}}\right)$ was significantly slower for 3 mutations (Error!

Reference source not found., Figure 2E).

No abnormalities were found in the fast time constant of inactivation ([.tau $\left.]_{\mathrm{F}}\right)$, late current $\left(\mathrm{I}_{\text {late }}\right)$, deactivation time constant $\left([\cdot \operatorname{tau}]_{\mathrm{D}}\right)$, and rate of recovery from inactivation $\left(\mathrm{t}_{1 / 2}\right)$.

\section{Correlation of Electrophysiologic Abnormalities with IBS Subtypes}

Channel properties that would lead to LOF in both channel activation and inactivation were most common (Error! Reference source not found., Figure 3A). Most of the LOF abnormalities were found in the four IBS-C subjects $(9 / 16=56 \%$, Figure $3 \mathrm{~B}$, red). Interestingly, the majority of LOF in IBS-C were loss in activation (5/8), suggesting that lack of $\mathrm{Na}_{V} 1.5$ activation may directly contribute to decreased activity in IBS-C. IBS-D subjects had a smaller number of abnormalities $(4 / 16=25 \%$, Figure $3 B$, blue). Unlike IBS$\mathrm{C}$, LOF parameters in IBS-D were mostly in $\mathrm{Na}_{\mathrm{V}} 1.5$ inactivation (3/4). It is presently 
unclear how loss of $\mathrm{Na}_{\mathrm{V}} 1.5$ inactivation may affect activity. Finally, only 2 functional abnormalities were discovered in IBS-M $(2 / 16=13 \%)$, with one being a GOF $\left(t_{\text {peak }}\right)$ and one being LOF $\left(\delta \mathrm{V}_{\mathrm{i}}\right)$ (Figure 3B, yellow).

\section{Clinical Case of Patient with A997T Nav1.5}

The most striking of our findings was a dramatically smaller, slower, and right-shifted $\mathrm{Na}^{+}$ current in A997T (Figure 2A, Figure 4A). This mutation belongs to a 65-year-old Caucasian female with Rome II IBS-C with lifelong constipation and intermittent abdominal pain and bloating, relieved by defecation. Previous medical history, ECG, laboratory testing, colonoscopy, defecating proctogram and anorectal manometry were unrevealing. A nuclear medicine, whole-gut transit study found normal gastric emptying and small bowel transit but a delay in colonic transit at 24 hours and 48 hours (Supplementary Figure 2).

Electrophysiology analysis showed that p.A997T-Nav1.5 channels are LOF phenotype, with abnormalities in multiple functional parameters (Error! Reference source not found.). Most obvious was a near complete abolition (98\% reduction) of peak current from $-151 \pm 27$ $\mathrm{pA} / \mathrm{pF}$ for wild-type $\mathrm{Na}_{\mathrm{V}} 1.5$ to $-2.3 \pm 0.5 \mathrm{pA} / \mathrm{pF}$ for p.A997T-Na 1.5 ( $\mathrm{n}=9-11, \mathrm{P}<0.05$, Figure 4A, B). Other abnormalities included a depolarized voltage dependence of activation $\left(\mathrm{V}_{1 / 2 \mathrm{a}}\right)$ of $-52.1 \pm 0.8 \mathrm{mV}$ versus $-58.2 \pm 1.0 \mathrm{mV}$, and a slower time-to-peak of $2.52 \pm 0.23 \mathrm{~ms}$ versus $1.43 \pm 0.04 \mathrm{~ms}$ (Figure 4C, D).

Mexiletine is known to rescue expression defects of $\mathrm{Na}_{\mathrm{V}} 1.5{ }^{32}$ We cultured HEK-293 cells that transiently expressed p.A997T-Na 1.5 in the presence of $10 \mu \mathrm{M}$ mexiletine for 48 hours and measured currents. Mexiletine treatment significantly enhanced p.A997T-Nav 1.5 current, increasing peak current 6-fold to $-15 \pm 4 \mathrm{pA} / \mathrm{pF}$ ( $\mathrm{n}=23-25, P<0.05$ compared to untreated p.A997T-Nav1.5 or treated wild-type, Figure 4A, B) and restored $\mathrm{V}_{1 / 2 \mathrm{a}}$ $(-52.1 \pm 0.8 \mathrm{mV})$ and time to peak $(1.54 \pm 0.12 \mathrm{~ms}, \mathrm{n}=12-17, \mathrm{P}<0.05$ vs. untreated A997T and $\mathrm{P}>0.05$ vs. wild-type) (Figure 4C, D).

Mexiletine rescue of p.A997T-Nav1.5 function in-vitro led us to hypothesize that this drug would also restore in-vivo colonic function. Prior to drug administration, the patient documented 5 complete spontaneous bowel movements over 3.5 weeks (1.4 $\pm 0.5 /$ week) and 5 small hard bowel movements (1.4 $\pm 1.0 /$ week) (Figure 5). Mexiletine was then administered orally in increasing doses from $200 \mathrm{mg}$ to $400 \mathrm{mg}$ every eight hours over 5 days while the patient was on continuous telemetry. An increase in QTc interval from study beginning to end (from $441 \mathrm{~ms}$ to $471 \mathrm{~ms}$ ) was not clinically significant. At the higher doses of mexiletine (300 - $400 \mathrm{mg}$ ), the patient experienced known central side effects including nausea, lightheadedness, and mild ataxia. However, except for missing one dose she was able to complete the study. Whole-gut transit after day 5 showed an increase in the rate of gastric emptying ( $\mathrm{t}_{50}$ from $132 \mathrm{~min}$ to $91 \mathrm{~min}$ ). The patient had 2 spontaneous bowel movements during the transit test but no change in colonic transit was found, likely reflecting passage of unlabeled stool from the left colon. Over a 5 week follow-up postmexiletine, the patient reported 25 complete spontaneous bowel movements $(5 \pm 2.0 /$ week, $\mathrm{P}<0.05$ compared to pre-mexiletine) and 2 hard small bowel movements $(0.4 \pm 0.5 /$ week, $\mathrm{P}>0.05$ compared to pre-mexiletine). This effect tapered over the 5 weeks off mexiletine (Figure 5). 


\section{Discussion}

\section{Distinctive SCN5A Variations in a Subset of IBS Patients}

A number of IBS-related putative genes have been identified, but almost all remain to be validated and at most contribute to the pathophysiology in 1-5\% of patients. ${ }^{33}$ Patients with SCN5A mutations predisposing to cardiac conduction disorders also have a higher prevalence of IBS. ${ }^{20}$ A pilot study of 49 patients suggested a $2.0 \%$ prevalence of SCN5A mutations in IBS. ${ }^{21}$ In this 584 patient cohort (Error! Reference source not found.) we confirm a $2.2 \%$ prevalence of $S C N 5 A$ mutations in IBS subjects (Figure 1). In particular, the newly identified mutations were absent from 2760 control alleles from Mayo blood donors, as well as from several large exomes databases (additional 7595 samples) that have become publicly available after the initiation of this study $\left(\mathrm{NHLBI}^{34}\right.$ and 1000 Genomes $^{35}$ ), with the exceptions of T220I, G615E and T1304M, which remain extremely rare among Caucasians (Supplementary Table 2).

We also discovered that an additional $2.9 \%$ have previously known, functionally relevant SCN5A coding variants (Supplementary Table 2). These results suggest that SCN5A status may play a role in $1.7 \%$ (electrophysiologically abnormal mutations) to $5.1 \%$ (mutations plus other coding polymorphisms) of IBS patients. Therefore, if $15 \%$ IBS prevalence is assumed, ${ }^{1}$ SCN5A coding abnormalities may contribute to IBS pathophysiology in up to 2.3 million IBS patients in the US. In addition to these, our GWAS-replication data suggest that common, less-damaging SCN5A variants and SCN5A transcriptional control may be relevant for IBS patients (Supplementary Table 5 and Supplementary Figures $3 \& 4$ ).

\section{IBS-related SCN5A Mutations Result in Functionally Abnormal Nav1.5 Channels}

Cardiology literature analyzing $S C N 5 A$ variants reveals that not all mutations are functionally relevant for the heart. ${ }^{36,37}$ Thus, functional analysis was required. Three mutations were identified and functionally characterized elsewhere, and our findings agreed with the previous report for T220I, ${ }^{29}$ but in contrast to previous reports, there was a lack of abnormal findings for T1304M, ${ }^{27}$ and a different set of functional abnormalities for G615E. ${ }^{31}$ These differences were likely secondary to the stringent statistical cutoff $(\mathrm{P}<0.01)$ for functional abnormalities used in this study and the GI focused protocols used versus the cardiac protocols used in previous studies.

We subdivided functional changes into two broad categories based on an expected increase (GOF) or decrease (LOF) of $\mathrm{Na}^{+}$flux and showed that $90 \%$ of the abnormal parameters could be classified as LOF. For the novel mutations, the majority of LOF abnormalities displayed a decrease in the slope of steady-state voltage-dependence of inactivation (46\%, Figure 2B \& C) and of the kinetics of activation (30\%) and inactivation (23\%) (Figure 2D \& $\mathrm{E})$. The decrease in the slope of the voltage-dependence of inactivation $\left(\delta \mathrm{V}_{\mathrm{i}}\right)$ would predict a smaller window current (Figure $2 \mathrm{C}$ ) and diminish availability of $\mathrm{Na}^{+}$current. Furthermore, the decreases in both $\delta \mathrm{V}_{\mathrm{i}}$ and time to peak ( $\mathrm{t}_{\text {peak }}$ ) would contribute to the decrease in the upstroke velocity of the slow waves.

Consistent with the LOF phenotype in-vitro, it appears that SCN5A mutations may predominantly result in IBS-C. Most of the LOF abnormalities were found in mutation 
positive subjects with IBS-C (Figure 3), which were enriched (31\%) compared to the overall cohort (10\%) (Error! Reference source not found.). It is currently unclear how the LOF abnormalities could yield a predominantly diarrhea phenotype in the mutation positive IBSD subjects, and this will require further study.

These data are also consistent with prior reports. $\mathrm{Na}_{\mathrm{V}} 1.5$ channels are found in human $\mathrm{ICC}^{14}$ and smooth muscle cells ${ }^{10,13}$, and blockade of the $\mathrm{Na}_{\mathrm{V}}$ channels decreases excitability: it hyperpolarizes the resting potential, ${ }^{14}$ slows upstroke and prolongs slow wave, ${ }^{38}$ and decreases frequency. ${ }^{14}$ Further, recent clinical studies show that a novel $\mathrm{Na}_{\mathrm{V}} 1.5$ blocker ranolazine is associated with significant constipation. ${ }^{16}$ Thus, our findings have implications for involvement of $\mathrm{Na}_{\mathrm{V}} 1.5$ in pathophysiology of gastrointestinal motility IBS.

\section{Nav1.5-A997T Abnormalities are Rescued in-vitro and in-vivo by Mexiletine}

As a proof-of-principle, we performed a detailed evaluation of one IBS-C patient with a Nav1.5-A997T mutation. Of the mutations studied, A997T exhibited significant changes in several parameters, with the most noticeable being a $90 \%$ loss of peak current when compared to controls. Our in-vitro model demonstrated that short term treatment with mexiletine reversed many of the A997T-Nav1.5 defects (Figure 4), consistent with previous reports of other defective $\mathrm{Na}_{\mathrm{V}} 1.5$ channels. ${ }^{32}$ Prompted by the in-vitro response, we proceeded with a clinical trial of mexiletine in this patient with IBS-C. After a 5-day treatment with mexiletine, the patient's bowel movement frequency increased to normal range. Consistent with a likely effect on regulation of expression, the resulting normalization of this patient's GI motility persisted for 5 weeks (Figure 5). Mexiletine carries a risk of cardiac arrhythmia ${ }^{39}$ making it unlikely to be FDA approved as an IBS therapy. However, these data do suggest that specific $\mathrm{Na}_{\mathrm{V}} 1.5$ and other ion channelopathies may benefit from targeted and individualized therapies.

\section{SCN5A Genotype-phenotype Relationship May be Organ-or Sex-specific}

The A997T mutation identified in our patient would result in an almost complete abolition of channel function and a predicted loss of $\sim 50 \%$ of the Nav 1.5 current in not only the GI tract but also in the cardiac myocytes. The loss of one SCN5A allele can manifest as significant cardiac electrophysiologic abnormalities, particularly in men. ${ }^{40}$ Our patient was a female and did not have a personal or family history of cardiac rhythm disturbances or ECG abnormalities before or during this study. It is interesting that despite equal heritability between the sexes, there is a striking 9:1 M:F predominance of cardiac expressivity in subjects with type 1 Brugada syndrome, ${ }^{41}$ which is in contrast to the 1:2 M:F IBS prevalence. ${ }^{42}$ Indeed, this IBS cohort was predominately female (85\%), consistent with known IBS epidemiology. ${ }^{42}$ Yet, in the two mutation-positive male subjects, there were more ECG findings than in the rest of the cohort, and both of these SCN5A mutations (I94V, P648L) exhibited LOF changes. Thus, a hypothesis that requires further scrutiny is that loss of $\mathrm{Na}_{\mathrm{V}} 1.5$ function principally manifests with a cardiac phenotype in males but a GI phenotype in females. 


\section{Strengths and Limitations}

The strengths of this study are: (1) a large mutation-discovery sample size of 584 patients with IBS, (2) independent evidence of SCN5A association with IBS in a GWAS and replication study, (3) functional assessment of the discovered mutations, and (4) proof-ofprinciple study in a patient with a LOF mutation, with normalization of her bowel habits using a drug we showed in vitro to normalize the $\mathrm{Na}_{\mathrm{V}}$ channel defect. As any study, there are limitations including the open labeled nature of the proof of concept study. This study will need to be repeated in another cohort, and there remains the outstanding need to examine in more detail the phenotypes with respect to organ and patient symptoms.

\section{Clinical Implications}

In the dawn of the personalized medicine era we are discovering that for genetically complex diseases such as IBS there may be cohorts of patients with well-defined genetic abnormalities. Ion channels are directly involved in the mechanisms of visceral pain and GI motility, therefore ion channelopathies may be involved in pathogenesis of IBS. For the first time we provide the direct molecular and functional evidence for functionally significant SCN5A mutations in a subset of IBS patients. As we demonstrate in a proof-of-concept study for one of these patients, Nav1.5 dysfunction may underlie the IBS pathogenesis and provide personalized treatment options for this subset of patients. In conclusion, the data suggest that a subset of patients with IBS may have an SCN5A-encoded Nav1.5 ion channelopathy which may represent a novel pathophysiologic mechanism and provide novel therapeutic options.

\section{Acknowledgments}

We thank Kristy Zodrow for secretarial assistance. This work was supported by NIH grants DK52766, the Mayo Clinic Center for Cell Signaling in Gastroenterology (NIDDK P30DK084567), the Mayo Clinic Windland Smith Rice Comprehensive Sudden Cardiac Death Program, and the Swedish Research Council (VR 2010-2976).

Grant Support: This work was supported by NIH grants DK52766 (GF), the Mayo Clinic Center for Cell Signaling in Gastroenterology (NIDDK P30DK084567), and the Mayo Clinic Windland Smith Rice Comprehensive Sudden Cardiac Death Program, and the Swedish Research Council (VR 2010-2976).

\section{Abbreviations}
IBS irritable bowel syndrome
ICC interstitial cells of Cajal

\section{References}

1. Drossman DA, Li ZM, Andruzzi E, et al. United-States Householder Survey of Functional Gastrointestinal Disorders - Prevalence, Sociodemography, and Health Impact. Dig Dis Sci. 1993; 38:1569-1580. [PubMed: 8359066]

2. Ford AC, Talley NJ. IBS in 2010: Advances in pathophysiology, diagnosis and treatment. Nat Rev Gastroenterol Hepatol. 2011; 8:76-8. [PubMed: 21293507]

3. Saito YA, Petersen GM, Larson JJ, et al. Familial aggregation of irritable bowel syndrome: a family case-control study. Am J Gastroenterol. 2010; 105:833-41. [PubMed: 20234344]

4. Bengtson MB, Ronning T, Vatn MH, et al. Irritable bowel syndrome in twins: genes and environment. Gut. 2006; 55:1754-9. [PubMed: 17008364] 
5. Saito YA. The role of genetics in IBS. Gastroenterol Clin North Am. 2011; 40:45-67. [PubMed: 21333900]

6. Farrugia G. Ionic conductances in gastrointestinal smooth muscles and interstitial cells of Cajal. Annu Rev Physiol. 1999; 61:45-84. [PubMed: 10099682]

7. Beyder A, Farrugia G. Targeting ion channels for the treatment of gastrointestinal motility disorders. Therap Adv Gastroenterol. 2012; 5:5-21.

8. Akbar A, Walters JR, Ghosh S. Review article: visceral hypersensitivity in irritable bowel syndrome: molecular mechanisms and therapeutic agents. Aliment Pharmacol Ther. 2009; 30:42335. [PubMed: 19493256]

9. Muraki K, Imaizumi Y, Watanabe M. Sodium currents in smooth muscle cells freshly isolated from stomach fundus of the rat and ureter of the guinea-pig. J Physiol. 1991; 442:351-75. [PubMed: 1665861]

10. Holm AN, Rich A, Miller SM, et al. Sodium current in human jejunal circular smooth muscle cells. Gastroenterology. 2002; 122:178-87. [PubMed: 11781292]

11. Strege PR, Mazzone A, Kraichely RE, et al. Species dependent expression of intestinal smooth muscle mechanosensitive sodium channels. Neurogastroenterol Motil. 2007; 19:135-43. [PubMed: 17244168]

12. Xiong Z, Sperelakis N, Noffsinger A, et al. Fast Na+ current in circular smooth muscle cells of the large intestine. Pflugers Arch. 1993; 423:485-91. [PubMed: 8394569]

13. Ou Y, Gibbons SJ, Miller SM, et al. SCN5A is expressed in human jejunal circular smooth muscle cells. Neurogastroenterol Motil. 2002; 14:477-86. [PubMed: 12358675]

14. Strege PR, Ou Y, Sha L, et al. Sodium current in human intestinal interstitial cells of Cajal. Am J Physiol Gastrointest Liver Physiol. 2003; 285:G1111-21. [PubMed: 12893628]

15. Der-Silaphet T, Malysz J, Hagel S, et al. Interstitial cells of cajal direct normal propulsive contractile activity in the mouse small intestine. Gastroenterology. 1998; 114:724-36. [PubMed: 9516393]

16. Nash DT, Nash SD. Ranolazine for chronic stable angina. Lancet. 2008; 372:1335-41. [PubMed: 18929905]

17. Gellens ME, George AL Jr. Chen LQ, et al. Primary structure and functional expression of the human cardiac tetrodotoxin-insensitive voltage-dependent sodium channel. Proc Natl Acad Sci U S A. 1992; 89:554-8. [PubMed: 1309946]

18. Tfelt-Hansen J, Winkel BG, Grunnet M, et al. Inherited cardiac diseases caused by mutations in the Nav1.5 sodium channel. J Cardiovasc Electrophysiol. 2010; 21:107-15. [PubMed: 19845816]

19. Bezzina CR, Barc J, Mizusawa Y, et al. Common variants at SCN5A-SCN10A and HEY2 are associated with Brugada syndrome, a rare disease with high risk of sudden cardiac death. Nat Genet. 2013; 45:1044-9. [PubMed: 23872634]

20. Locke GR 3rd, Ackerman MJ, Zinsmeister AR, et al. Gastrointestinal symptoms in families of patients with an SCN5A-encoded cardiac channelopathy: evidence of an intestinal channelopathy. Am J Gastroenterol. 2006; 101:1299-304. [PubMed: 16771953]

21. Saito YA, Strege PR, Tester DJ, et al. Sodium channel mutation in irritable bowel syndrome: evidence for an ion channelopathy. Am J Physiol Gastrointest Liver Physiol. 2009; 296:G211-8. [PubMed: 19056759]

22. Ackerman MJ, Siu BL, Sturner WQ, et al. Postmortem molecular analysis of SCN5A defects in sudden infant death syndrome. JAMA. 2001; 286:2264-9. [PubMed: 11710892]

23. Kapplinger JD, Tester DJ, Alders M, et al. An international compendium of mutations in the SCN5A-encoded cardiac sodium channel in patients referred for Brugada syndrome genetic testing. Heart Rhythm. 2010; 7:33-46. [PubMed: 20129283]

24. Yang P, Kanki H, Drolet B, et al. Allelic variants in long-QT disease genes in patients with drugassociated torsades de pointes. Circulation. 2002; 105:1943-8. [PubMed: 11997281]

25. Kapplinger JD, Tester DJ, Salisbury BA, et al. Spectrum and prevalence of mutations from the first 2,500 consecutive unrelated patients referred for the FAMILION long QT syndrome genetic test. Heart Rhythm. 2009; 6:1297-303. [PubMed: 19716085] 
26. Wattanasirichaigoon D, Vesely MR, Duggal P, et al. Sodium channel abnormalities are infrequent in patients with long QT syndrome: identification of two novel SCN5A mutations. Am J Med Genet. 1999; 86:470-6. [PubMed: 10508990]

27. Wang DW, Desai RR, Crotti L, et al. Cardiac sodium channel dysfunction in sudden infant death syndrome. Circulation. 2007; 115:368-76. [PubMed: 17210841]

28. Makita N, Behr E, Shimizu W, et al. The E1784K mutation in SCN5A is associated with mixed clinical phenotype of type 3 long QT syndrome. J Clin Invest. 2008; 118:2219-29. [PubMed: 18451998]

29. Benson DW, Wang DW, Dyment M, et al. Congenital sick sinus syndrome caused by recessive mutations in the cardiac sodium channel gene (SCN5A). J Clin Invest. 2003; 112:1019-28. [PubMed: 14523039]

30. Gui J, Wang T, Jones RP, et al. Multiple loss-of-function mechanisms contribute to SCN5Arelated familial sick sinus syndrome. PLoS One. 2010; 5:e10985. [PubMed: 20539757]

31. Albert CM, Nam EG, Rimm EB, et al. Cardiac sodium channel gene variants and sudden cardiac death in women. Circulation. 2008; 117:16-23. [PubMed: 18071069]

32. Valdivia CR, Ackerman MJ, Tester DJ, et al. A novel SCN5A arrhythmia mutation, M1766L, with expression defect rescued by mexiletine. Cardiovasc Res. 2002; 55:279-89. [PubMed: 12123767]

33. Saito YA, Mitra N, Mayer EA. Genetic approaches to functional gastrointestinal disorders. Gastroenterology. 2010; 138:1276-85. [PubMed: 20176021]

34. Exome Variant Server, NHLBI GO Exome Sequencing Project (ESP). Seattle, WA: (URL: http:// evs.gs.washington.edu/EVS/) [12/25/2013 accessed]

35. Abecasis GR, Auton A, Brooks LD, et al. An integrated map of genetic variation from 1,092 human genomes. Nature. 2012; 491:56-65. [PubMed: 23128226]

36. Kapa S, Tester DJ, Salisbury BA, et al. Genetic Testing for Long-QT Syndrome: Distinguishing Pathogenic Mutations From Benign Variants. Circulation. 2009; 120:1752-1760. [PubMed: 19841300]

37. Mazzone A, Strege PR, Tester DJ, et al. A mutation in telethonin alters Nav1.5 function. J Biol Chem. 2008; 283:16537-44. [PubMed: 18408010]

38. Barajas-Lopez C, Den Hertog A, Huizinga JD. Ionic basis of pacemaker generation in dog colonic smooth muscle. J Physiol. 1989; 416:385-402. [PubMed: 2481730]

39. Velebit V, Podrid P, Lown B, et al. Aggravation and provocation of ventricular arrhythmias by antiarrhythmic drugs. Circulation. 1982; 65:886-94. [PubMed: 6176355]

40. Papadatos GA, Wallerstein PM, Head CE, et al. Slowed conduction and ventricular tachycardia after targeted disruption of the cardiac sodium channel gene Scn5a. Proc Natl Acad Sci U S A. 2002; 99:6210-5. [PubMed: 11972032]

41. Benito B, Sarkozy A, Mont L, et al. Gender differences in clinical manifestations of Brugada syndrome. J Am Coll Cardiol. 2008; 52:1567-73. [PubMed: 19007594]

42. Saito YA, Schoenfeld P, Locke GR 3rd. The epidemiology of irritable bowel syndrome in North America: a systematic review. Am J Gastroenterol. 2002; 97:1910-5. [PubMed: 12190153] 
DII

DIII

DIV

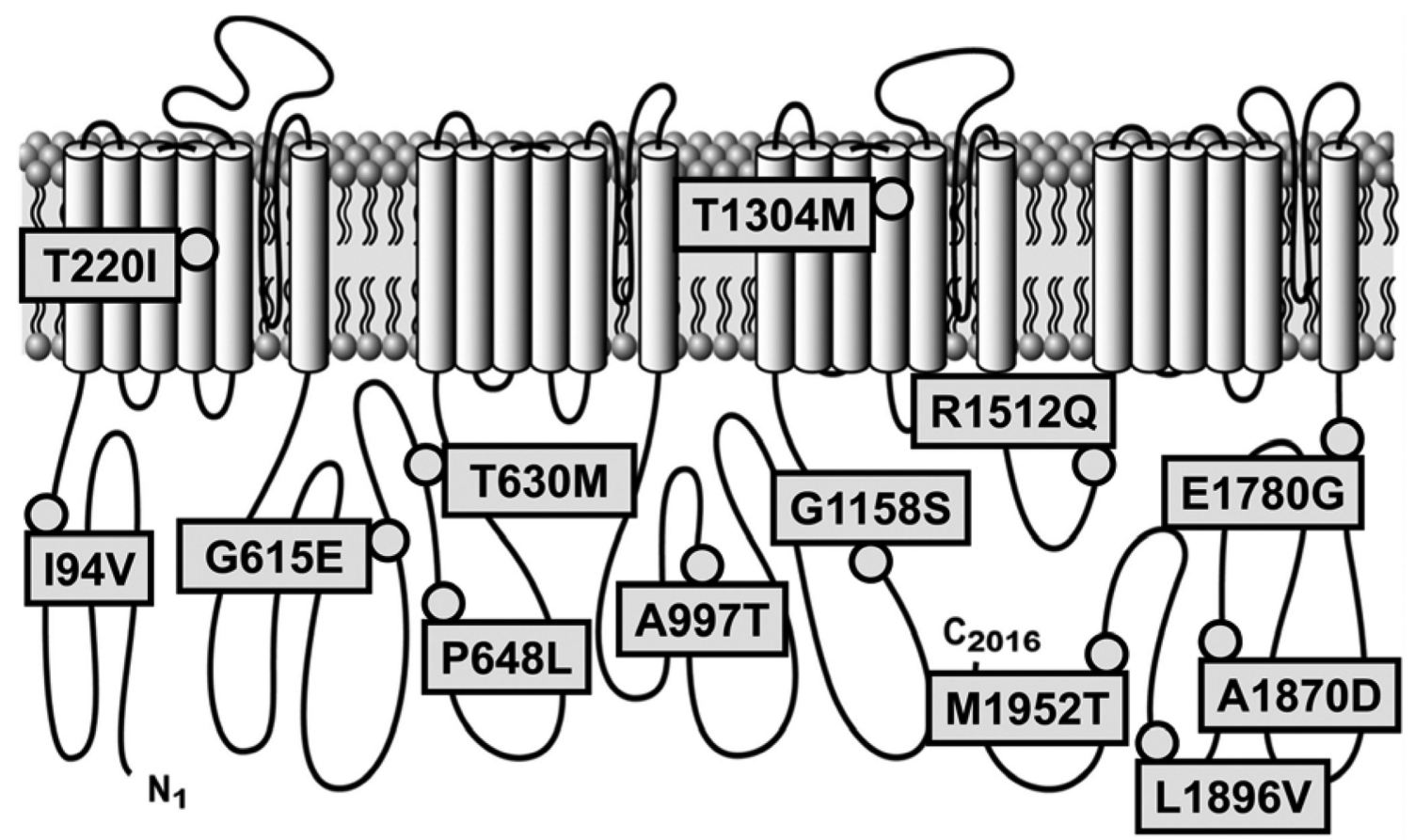

Figure 1.

Voltage-gated $\mathrm{Na}^{+}$ion channel $\mathrm{Na}_{\mathrm{V}} 1.5$ topology with missense mutations in $S C N 5 \mathrm{~A}$ identified in a cohort with IBS. DI - DIV are homologous 6 transmembrane helix domains 1 through 4. 

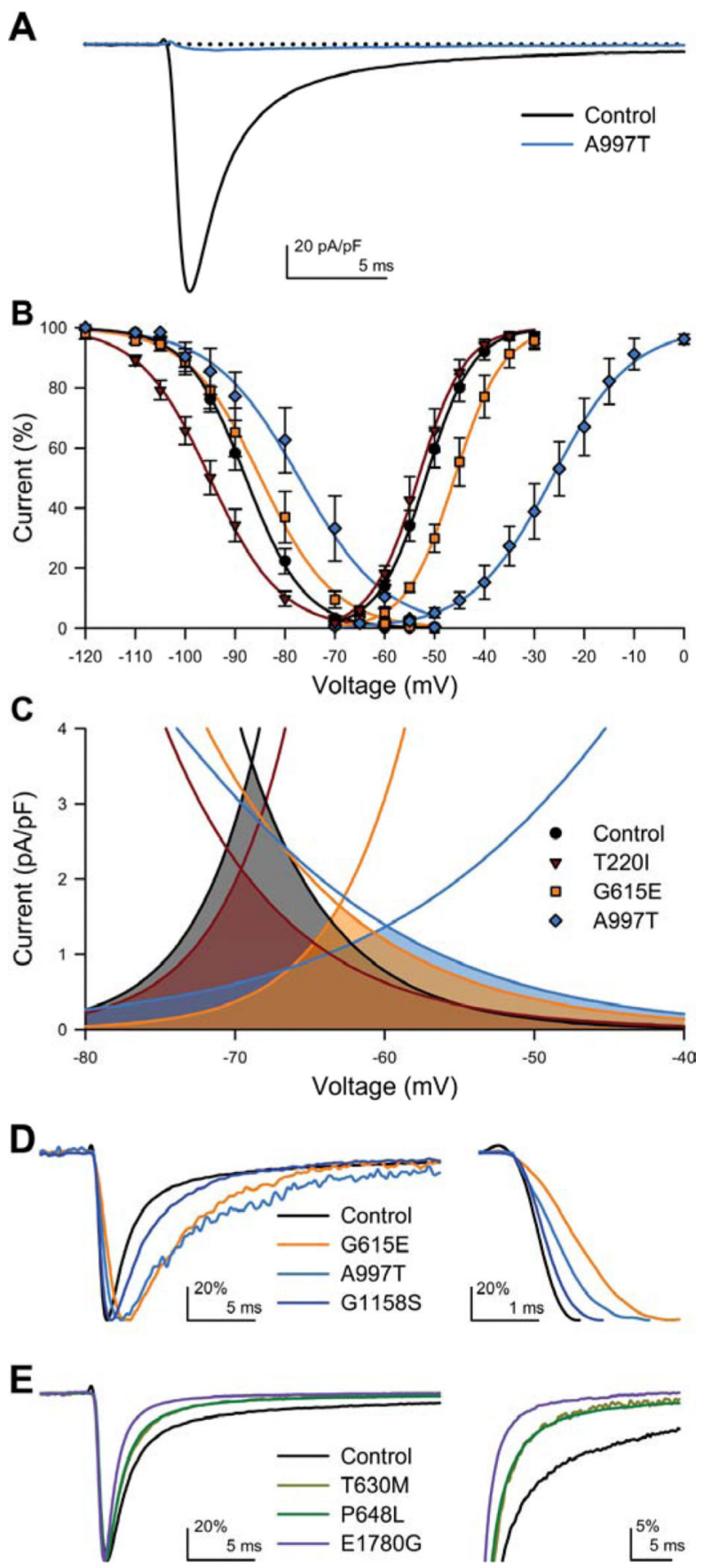

Figure 2.

Electrophysiologic abnormalities in $\mathrm{Na}_{\mathrm{V}} 1.5$ mutations. $A$, Representative whole cell $\mathrm{Na}^{+}$ current traces (step to $-30 \mathrm{mV}$ ) from HEK293 cells transfected with wild-type (control, black) or A997T (blue). Dotted line $=0 \mathrm{pA} / \mathrm{pF} . B, \mathrm{Na}_{\mathrm{V}} 1.5$ current-voltage plot showing shifts in voltage-dependence of inactivation for T220I (maroon) and A997T (blue) and positive shifts in voltage-dependence of activation for G615E (orange) and A997T (blue). $C$, Window currents for T220I, G615E, A997 are the shaded areas under the intersecting current-voltage curves. $D \& E$ show representative single traces (step to $-30 \mathrm{mV}$, peaks 
normalized to $100 \%$ ) of whole cell $\mathrm{Na}^{+}$voltage-dependent current for mutations compared to wild-type (controls) for in the activation $(D)$ and inactivation kinetics $(E) . D$, Activation kinetics were altered for mutations G615E (orange), A997T (blue), and G1158S (violet) compared to wild-type, (control, black). Inset, G615E, A997T, and G1158S activate slower than wild-type. $E$, Inactivation kinetics were altered for mutations T630M (maize), P648L (green), or E1780G (purple) compared to wild-type (control, black). Inset, mutations T630M, P648L, or E1780G inactivate faster than wild-type. 

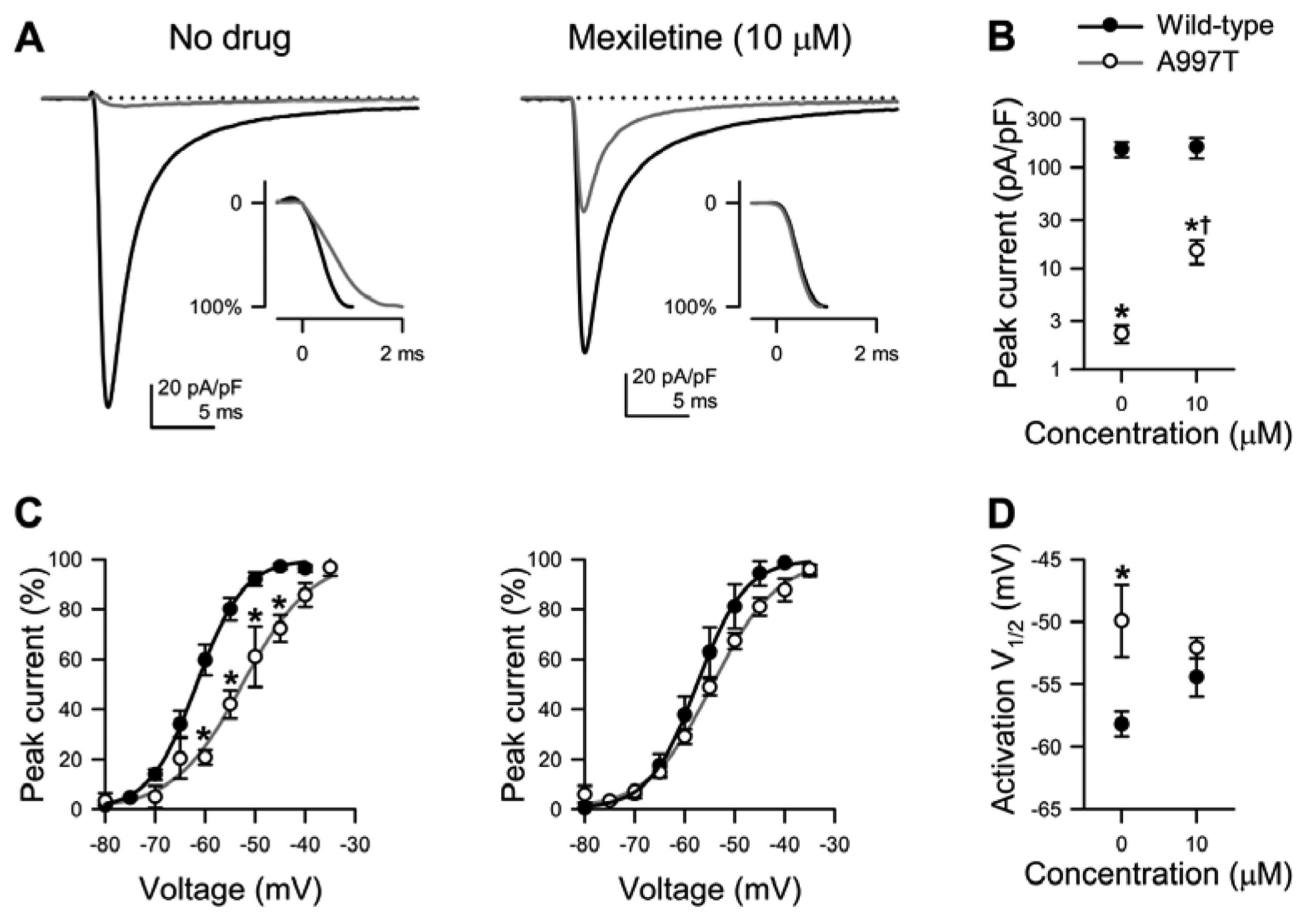

Figure 3.

Distribution of the electrophysiologic abnormalities across all mutations. $A$, Shown in black are gain-of-function (GOF, 2/18) and in grey are loss-of-function (LOF, 16/18) parameters for all IBS cases (IBS-D, IBS-C and IBS-M). $B$, Activation and inactivation LOF abnormalities are shown in IBS-D (4/18, blue), IBS-C (8/16, red) and IBS-M (1/16, yellow). 

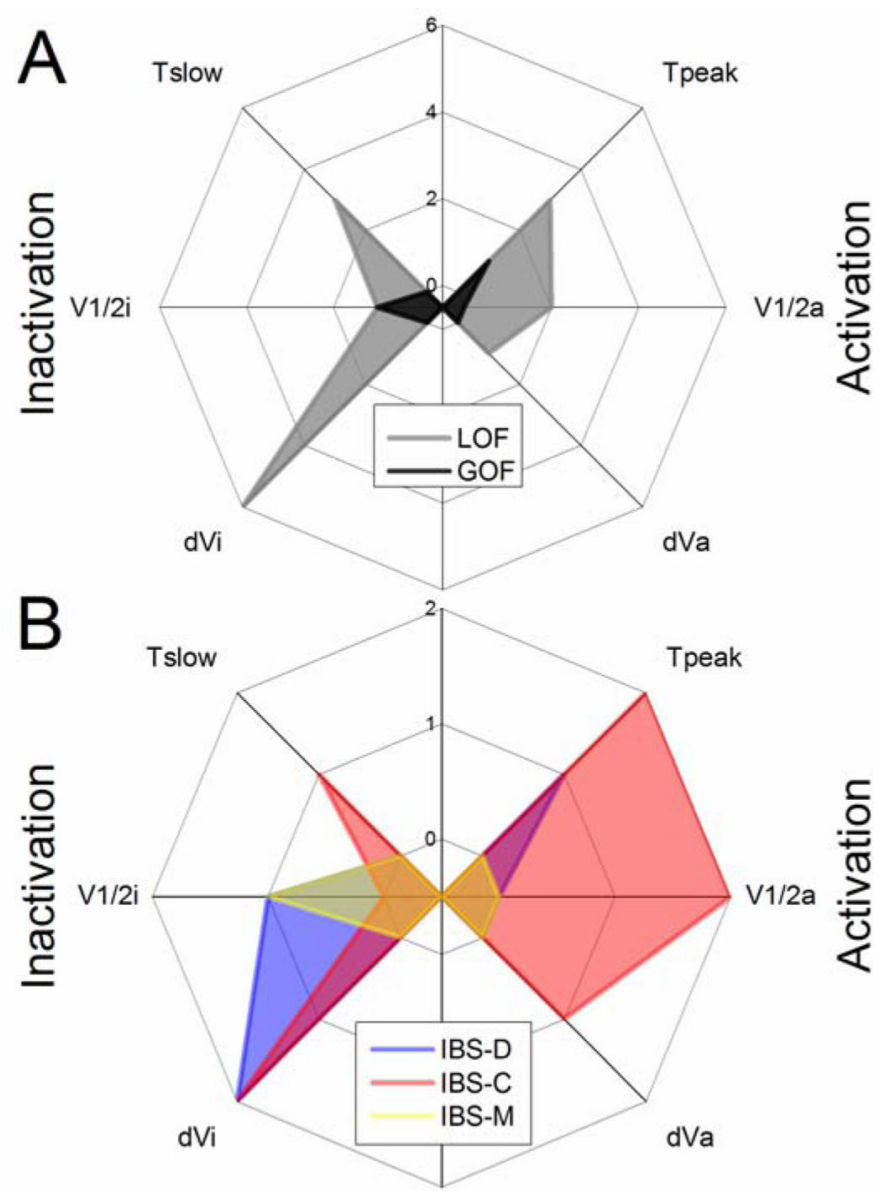

Figure 4.

Loss-of-function phenotype in the mutant sodium channel p.A997T-Nav1.5 with partial rescue by mexiletine. $A$, Representative $\mathrm{Na}^{+}$current traces (step to $-30 \mathrm{mV}$ ) from wild-type (black trace) or p.A997T-Na 1.5 (grey trace) without (left) or with (right) mexiletine $(10 \mu \mathrm{M})$. Inset shows the same traces scaled to peak. $B$, Averages of peak current densities. $* \mathrm{P}<0.05$ to wild-type and $\dagger \mathrm{P}<0.05$ to no drug by a one-way parametric ANOVA with Bonferroni's posttest $(\mathrm{n}=9-25)$. $C$, Voltage dependence of steady-state activation and inactivation of wild-type (filled symbols) or A997T-Nav1.5 (empty symbols) previously exposed to no drug (left) or mexiletine (right). Boltzmann function fits for wild-type Nav1.5 (black lines) or A997T-Na 1.5 (grey lines). $D$, Averages of $\mathrm{V}_{1 / 2}$ of activation. ${ }^{*} \mathrm{P}<0.05$ to wild-type by a oneway parametric ANOVA with Bonferroni's posttest $(n=9-25)$. 


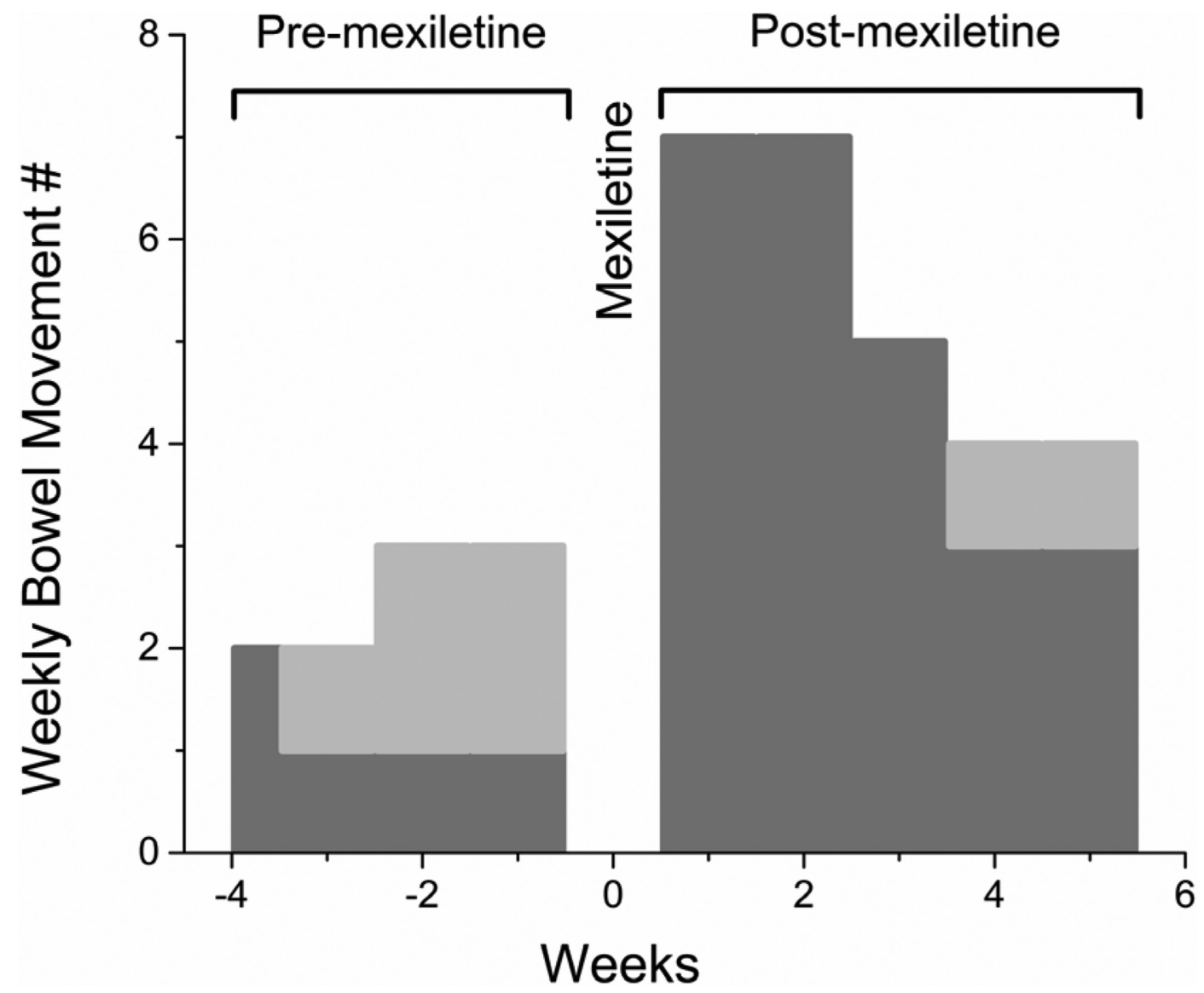

Figure 5.

Bowel movement frequency was improved in the A997T-Nav1.5 patient after mexiletine treatment. Complete spontaneous (dark grey) and small hard (light grey) bowel movements for the patient for 3.5 weeks preceding and 5 weeks following a 5 day treatment with mexiletine showing normalization of bowel habits with mexiletine. 


\section{Table 1}

Patient cohort characteristics.

\begin{tabular}{lcc}
\hline & IBS Cases n = 584 & IBS Cases with SCN5A Mutation n = 13 \\
\hline Median age (range) & $49.5(18.0-70.0)$ & $49.8(30.0-62.0)$ \\
\# Female (\%) & $484(83)$ & $11(85)$ \\
Race & & \\
Caucasian, n (\%) & $549(94)$ & $12(92)$ \\
Non-Caucasian, n (\%) & $35(6)$ & $1(8)$ \\
Not Stated, n (\%) & & \\
Region & $396(68)$ & $10(77)$ \\
Local, n (\%) & $3(23)$ \\
National, n (\%) & $187(32)$ & \\
Met Rome II criteria, n (\%) & $328(59)$ & $4(31)$ \\
IBS Subtype & & $3(23)$ \\
IBS-C, n (\%) & $59(10)$ & $3(23)$ \\
IBS-D, n (\%) & $143(25)$ & $3(23)$ \\
IBS-M, n (\%) & $181(31)$ & \\
Other, n (\%) & $201(34)$ & \\
\hline
\end{tabular}




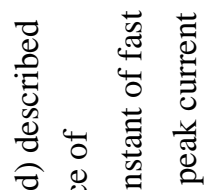

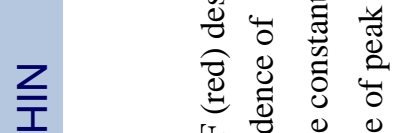

秀

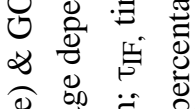

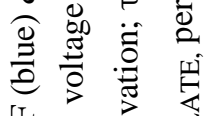

崩

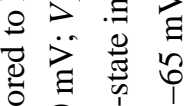

응 仓

泀芯

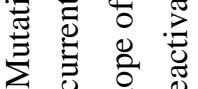

产苛

.0.0

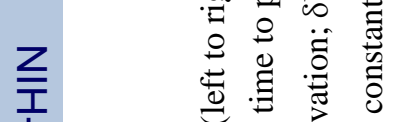

D $\quad$ o

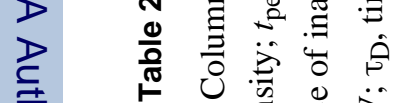

을

过苛苛

:

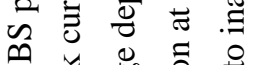

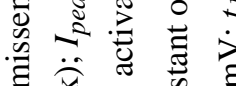

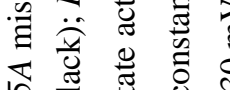

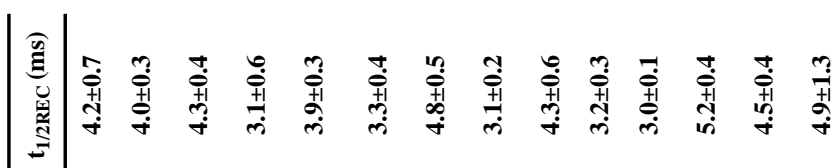

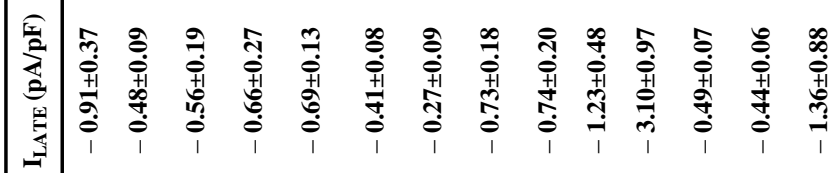

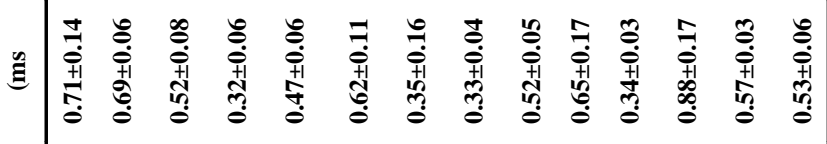

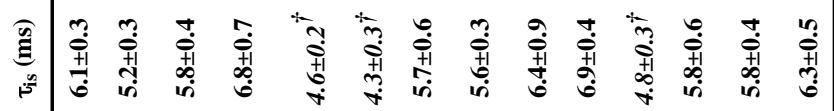

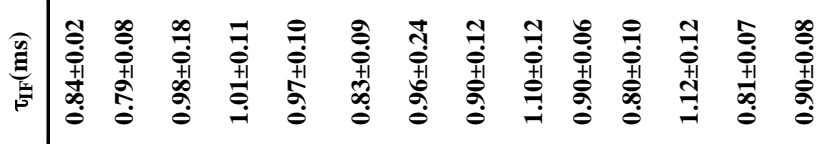

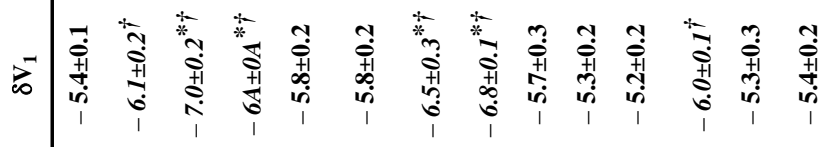

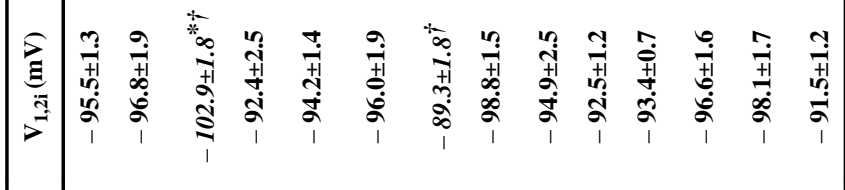

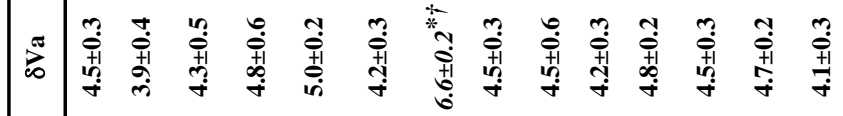

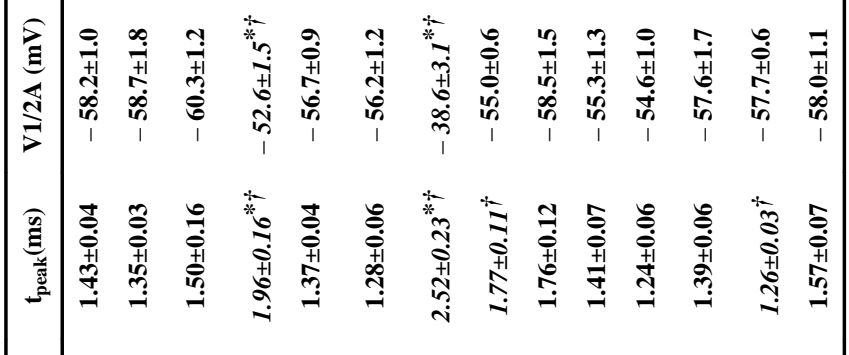

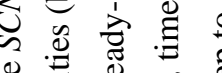

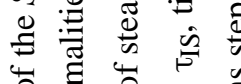

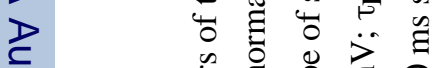

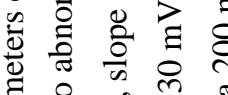

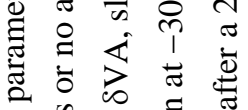

$\frac{\text { ำ }}{\frac{2}{2}}$

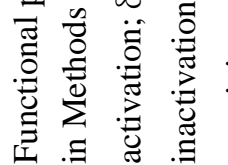

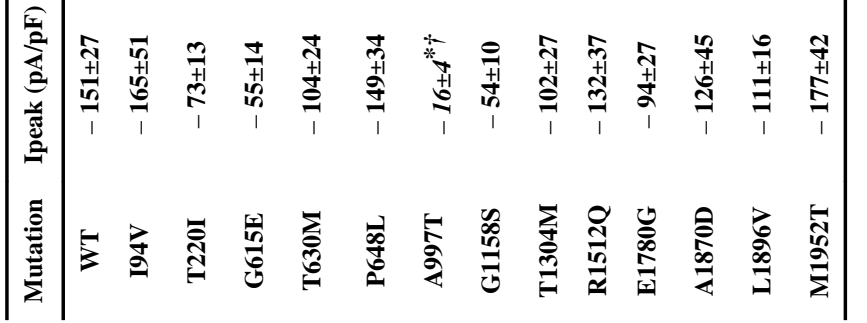

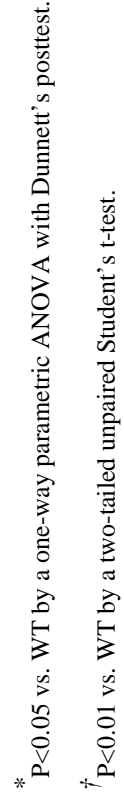

